\title{
DMOG, a Prolyl Hydroxylase Inhibitor, Increases Hemoglobin Levels without Exacerbating Hypertension and Renal Injury in Salt-Sensitive Hypertensive Rats
}

\author{
Sota Kato, Teisuke Takahashi, Noriyuki Miyata, and Richard J. Roman \\ Department of Pharmacology and Toxicology, University of Mississippi Medical Center, Jackson, Mississippi (S.K., R.J.R.); and \\ Pharmacology Laboratories (S.K., T.T.) and Research Headquarters of Pharmaceutical Operation (N.M.), Taisho Pharmaceutical \\ Co., Ltd., Saitama, Japan.
}

Received September 19, 2019; accepted November 25, 2019

\begin{abstract}
Prolyl hydroxylase (PHD) inhibitors are being developed as alternatives to recombinant human erythropoietin (rHuEPO) for the treatment of anemia in patients with chronic kidney disease (CKD). However, the effects of PHD inhibitors and rHuEPO on blood pressure and CKD in animal models susceptible to hypertension and nephropathy have not been studied. The present study compared the effects of dimethyloxaloylglycine (DMOG), a PHD inhibitor, and rHuEPO on the development of hypertension and renal injury in Dahl salt-sensitive rats fed an $8 \%$ salt diet for 3 weeks. DMOG and rHuEPO were equally effective at raising hemoglobin levels. Systolic blood pressure rose to a greater extent in rHuEPO-treated rats $(267 \pm 10$ vs. $226 \pm 4 \mathrm{~mm} \mathrm{Hg})$ than in rats given DMOG $(189 \pm 8 \mathrm{~mm} \mathrm{Hg})$. Urinary protein excretion increased to $568 \pm 54$ versus $353 \pm$ $25 \mathrm{mg} /$ day in rats treated with rHuEPO and vehicle; however, it only rose to $207 \pm 21 \mathrm{mg} /$ day in rats receiving DMOG. DMOG significantly attenuated the degree of glomerulosclerosis and renal interstitial fibrosis as compared with that in vehicle and rHuEPO-treated rats. This was associated with lower renal levels
\end{abstract}

\section{Introduction}

Erythropoietin (EPO) acts on its receptor to activate the Janus-activating kinase 2 signaling cascade to stimulate the differentiation of erythroid progenitor cells into erythrocytes (Koury and Haase, 2015; Kuhrt and Wojchowski, 2015). EPO is produced in EPO-producing cells found in the renal cortical interstitium (Obara et al., 2008; Paliege et al., 2010). In chronic kidney disease (CKD), these cells transform into myofibroblasts and lose the ability to produce EPO (Asada et al., 2011; Souma et al., 2013). Consequently, patients with CKD develop anemia. The prevalence of anemia is $20 \%$ in patients with stage $3 \mathrm{CKD}$ and $60 \%$ and $75 \%$ in patients with stage 4 or stage 5 CKD (McFarlane et al., 2008).

This work was supported in part by the National Institutes of Health National Institute of Diabetes and Digestive and Kidney Diseases [Grant DK104184] and National Heart, Lung, and Blood Institute [Grant HL138685] and funds provided by Taisho Pharmaceutical Co., Ltd., Saitama, Japan.

https://doi.org/10.1124/jpet.119.262782. of monocyte chemoattractant protein- 1 and interleukin- $1 \beta$ and increased vascular endothelial growth factor expression in cortex and medulla. These results indicate that DMOG and rHuEPO are equally effective in increasing hemoglobin levels in Dahl S rats; however, rHuEPO aggravates hypertension and renal injury, whereas DMOG has marked renoprotective effects. These results suggest that $\mathrm{PHD}$ inhibitors may have a therapeutic advantage for the treatment of anemia in CKD.

\section{SIGNIFICANCE STATEMENT}

Prolyl hydroxylase (PHD) inhibitors are in phase 3 clinical trials as alternatives to recombinant human erythropoietin ( $\mathrm{rHuEPO}$ ) for the treatment of anemia in chronic kidney disease (CKD). The present study reveals that dimethyloxaloylglycine (DMOG), a PHD inhibitor, and rHuEPO are equally effective in increasing hemoglobin levels in Dahl S rats; however, rHuEPO aggravated hypertension and renal injury, whereas DMOG attenuated the development of hypertension and prevented renal injury. PHD inhibitors may provide a safer therapeutic option for the treatment of anemia in CKD.

Recombinant human EPO (rHuEPO) is widely used to compensate for the deficiency in EPO production to treat anemia and improves the quality of life in patients with CKD (Evans et al., 1990). On the other hand, some issues are associated with the use of rHuEPO for the treatment of anemia in patients with CKD. rHuEPO is given by chronic injections and is painful for nondialysis patients. Chronic administration of exogenous rHuEPO promotes the development of anti-rHuEPO antibodies, which neutralizes endogenous EPO (Means, 2016). Treatment of rHuEPO has also been reported to promote the development of hypertension, perhaps by increasing blood viscosity (Letcher et al., 1981; Raine, 1988; Steffen et al., 1989). Furthermore, three pivotal clinical studies have raised concerns about the safety of high-dose rHuEPO for the treatment of anemia. In the CREATE and the TREAT studies, the risks of hypertension, dialysis, and stroke were higher when the target hemoglobin level was high (Drüeke et al., 2006; Pfeffer et al., 2009). In the CHOIR study, high doses of rHuEPO increased the risk of

ABBREVIATIONS: BUN, blood urea nitrogen; CKD, chronic kidney disease; DMOG, dimethyloxaloylglycine; EPO, erythropoietin; HIF, hypoxiainducible factor; IL-1 $\beta$, interleukin- $\beta$; KIM-1, kidney injury marker 1 ; NAG, $N$-acetyl- $\beta$-D-glucosaminidase; PHD, prolyl hydroxylase; rHuEPO, recombinant human EPO; TGF $\beta 1$, transforming growth factor $\beta 1$. 
cardiovascular events independent of the target hemoglobin level (Singh et al., 2006; Szczech et al., 2008).

Potential alternatives to $\mathrm{rHuEPO}$ for the treatment of anemia are prolyl hydroxylase (PHD) inhibitors. PHD hydroxylates proline residues of hypoxia-inducible factor $\alpha(\mathrm{HIF} \alpha)$, which is a master regulator of the hypoxic response (Epstein et al., 2001). Hydroxylated HIF $\alpha$ is recognized by a ubiquitin ligase, von Hippel-Lindau protein, and degraded through the ubiquitin-proteasome pathway (Jaakkola et al., 2001). In hypoxic conditions, the PHD activity is reduced, and $\mathrm{HIF} \alpha$ escapes from hydroxylation and subsequent degradation. Once HIF $\alpha$ is stabilized, HIF $\alpha$ binds to the hypoxia response element together with cAMP response element binding protein (CREB) binding protein /p300 and constitutively active $\mathrm{HIF} \beta$ to upregulate the expression of target genes (Haase, 2006). As a consequence, vascularity is increased through HIF $1 \alpha$, and hemoglobin levels are elevated through the actions of HIF2 $\alpha$ (Elson et al., 2001; Percy et al., 2008). PHD inhibitors are under the investigation in ongoing phase 3 clinical trials to treat renal anemia in both nondialysis and dialysis patients (Cernaro et al., 2019). Recently, roxadustat was approved for the treatment of anemia in CKD in China (Dhillon, 2019). PHD inhibitors are orally active (Flamme et al., 2014; Kato et al., 2018) and upregulate the formation of endogenous EPO and other renoprotective factors such as vascular endothelial growth factor (VEGF). Therefore, it is important to compare the safety profile of $\mathrm{PHD}$ inhibitors and rHuEPO in models of renal disease.

Several studies have investigated the effects of rHuEPO on renal function in several CKD models in normotensive strains of rats and mice (Lee et al., 2005; Katavetin et al., 2007; Toba et al., 2009; Cañadillas et al., 2010; Rjiba-Touati et al., 2012). In these studies, $\mathrm{rHuEPO}$ reduced renal inflammation and fibrosis regardless of hematopoietic effect. On the other hand, hypertension and diabetes are the primary risk factors for the development of CKD, and these patients may be more susceptible to potential adverse effects of $\mathrm{rHuEPO}$ on blood pressure and hypertension-induced renal injury. To explore this possibility, we compared the effects of rHuEPO and dimethyloxaloylglycine (DMOG), an injectable PHD 1/2/3 pan inhibitor (Epstein et al., 2001), on the development of hypertension and renal injury in Dahl S rats, which are highly susceptible to the development of salt-sensitive hypertension and renal injury. This study demonstrated that DMOG markedly attenuated the development of hypertension and renal injury in this model, whereas rHuEPO had the opposite effect. These findings suggest that PHD inhibitors may provide a safer therapeutic option for the treatment of anemia in diabetes- and hypertension-induced CKD.

\section{Materials and Methods}

General. DMOG was synthesized by Medical Chemistry Laboratories in Taisho Pharmaceutical Co., Ltd. rHuEPO (PROCRIT) was purchased from Centocor Ortho Biotech Products, L.P. These experiments were performed using male Dahl S rats obtained from inbred colonies maintained in the University of Mississippi Medical Center. They were maintained on a $0.4 \%$ salt diet from weaning to the start of the experiments. All the experiments were approved by the Animal Care Committee of the University of Mississippi Medical Center.

Effect of DMOG on EPO Expression. These experiments were performed on male 9 -week-old Dahl S rats. A control blood sample was collected from the jugular vein, and the rats received intraperitoneal injection of saline or DMOG at a dose of $600 \mathrm{mg} / \mathrm{kg}$. Blood samples were collected from jugular vein 4,8 , and 24 hours later. The samples were centrifuged at $2130 \mathrm{~g}$ for 10 minutes, and serum EPO levels were measured using an ELISA kit (R\&D Systems, Minneapolis, MN). Additional groups of rats were given DMOG $600 \mathrm{mg} / \mathrm{kg}$, and the kidneys were removed before ( 0 hours) and 1, 2, and 4 hours after administration. mRNA was extracted from the renal cortex using TRIZOL (Life Technologies, Grand Island, NY). The samples were reverse transcribed using a poly $\mathrm{t}$ and random hexamer primers. EPO mRNA levels were determined using quantitative reverse transcription-polymerase chain reaction. EPO was amplified using the following forward and reverse primers: 5-GCTCCAATCTTTGTG GCATCT-3 and 5-TGGCTTCGTGACCCTCTGT-3. $\beta$-Actin forward and reverse primers were 5-CCTCTATGCCAACACAGTGC-3 and 5GTACTCCTGCTTGCTGATCC-3.

Time Course of the Effects of DMOG and rHuEPO. Male 9week-old Dahl S rats were randomly assigned to four groups and treated with vehicle, DMOG at $60 \mathrm{mg} / \mathrm{kg}$, DMOG at $600 \mathrm{mg} / \mathrm{kg}$, or $\mathrm{rHuEPO}$ at $100 \mathrm{U} / \mathrm{kg}$. DMOG was dissolved in $0.9 \% \mathrm{NaCl}$ solution, and $\mathrm{rHuEPO}$ was diluted with a $0.5 \% \mathrm{BSA}$ in a $0.9 \% \mathrm{NaCl}$ solution. DMOG was given intraperitoneally, whereas $\mathrm{rHuEPO}$ was given subcutaneously, three times per week starting from day 1 .

Baseline urinary protein excretion, blood pressure, hemoglobin, and hematocrit were measured during a control period while the rats were fed a normal-salt diet containing $0.4 \% \mathrm{NaCl}$. Then, they were switched to a high-salt diet $(8.0 \% \mathrm{NaCl})$, DMOG or EPO was administered for 3 weeks, and samples were collected weekly. Urine samples were collected using metabolic cages, and urine protein concentrations were determined by Bradford method (Bio-Rad Laboratories, Hercules, CA). Blood pressure was measured using a tail-cuff device (Hatteras Instruments, Cary, NC). Hemoglobin levels were determined in $10 \mu \mathrm{l}$ of blood using HemoCue $\mathrm{Hb} \mathrm{201+}$ device (HemoCue, Brea, CA), and hematocrit was determined in $80-\mu l$ blood samples collected in a hematocrit tube from tail vein.

Histology. After 3 weeks of treatment with vehicle, DMOG, or $\mathrm{rHuEPO}$, the rats were sacrificed, and the left kidneys were fixed in $10 \%$ buffered formalin. Paraffin sections $(3 \mu \mathrm{m})$ were stained with Masson's trichrome and analyzed for the degree of glomerulosclerosis and glomerular and renal interstitial fibrosis. Glomerulosclerosis was scored on a scale from 0 to 4 , where 0 represents a normal glomerulus, 1 represents 1\%-25\% loss of capillary area, 2 represents 26\%-50\% loss, 3 represents $51 \%-75 \%$ loss, and 4 represents $>75 \%$ loss. The images were captured using a Nikon Eclipse 55i microscope equipped with a Nikon DS-Fi1 color camera (Nikon Instruments Inc., Melville, NY). The degree of renal interstitial fibrosis was calculated as the percentage of area stained blue using the NIS Elements 3.0 software (Nikon Instruments Inc.). Protein casts were determined as the percentage of area stained red in the sections (Muroya et al., 2015).

Measurement of Renal Injury Biomarkers. Blood urea nitrogen (BUN) levels were determined using a BUN detection kit (Arbor Assays, Ann Arbor, MI). Serum creatinine levels were determined using a LabAssay Creatinine kit (Wako Pure Chemical Industries, Osaka, Japan). Urinary $N$-acetyl- $\beta$-D-glucosaminidase (NAG) and kidney injury marker 1 (KIM-1) were determined using a NAG assay kit (SIGMA, St. Louis, MO) and a rat KIM-1 ELISA kit (R\&D Systems).

Measurement of Renal Inflammatory Markers and VEGF Levels. The right kidneys were separated into cortex and medulla, and they were homogenized in a Tris buffer containing $5 \mathrm{mM}$ EDTA, $1 \mathrm{mM}$ EGTA, $1 \%$ Triton X-100, and a proteinase inhibitor cocktail. The homogenate was centrifuged at $11,000 \mathrm{~g}$ for 5 minutes at $4^{\circ} \mathrm{C}$, and the monocyte chemoattractant protein-1 (MCP-1), interleukin-1 $\beta$ (IL-1 $\beta$ ), transforming growth factor $\beta 1$ (TGF $\beta 1$ ), and VEGF levels in the supernatant were measured using ELISA kits (R\&D Systems). Samples for measurement of TGF $\beta 1$ were first activated by acidification for 5 minutes and then measured using an ELISA kit from R\&D systems. 
Statistics. Data are presented as mean values \pm 1 S.E. The statistical significances of differences were determined using the Student's $t$ test for comparisons between two groups, a one-way ANOVA and Holm-Sidak test for multiple comparisons, or a two-way ANOVA for repeated measures followed by a Holm-Sidak test for the time course studies (Sigma Plot 11; Systat Software, San Jose, CA).

\section{Results}

Effect of DMOG on EPO Expression. We first addressed the effect of DMOG on renal EPO mRNA expression and serum EPO concentration after administration of $600 \mathrm{mg} / \mathrm{kg}$ of DMOG. EPO mRNA levels in the renal cortex started to increase 1 hour after the administration of DMOG. EPO mRNA levels at 4 hours were 70-fold higher than those seen at baseline (Fig. 1A). Serum EPO concentration in the vehicle-treated group was below detection 24 hours after administration. The EPO concentration in the $600 \mathrm{mg} / \mathrm{kg}$ of the DMOG-treated group increased to $890 \mathrm{pg} / \mathrm{ml}, 4$ hours after administration. The levels returned to the baseline 24 hours after the administration of DMOG (Fig. 1B).

Comparison of the Hematopoietic Effects of Chronic DMOG and rHuEPO Administration. The hematopoietic effects of DMOG (60 or $600 \mathrm{mg} / \mathrm{kg}$, i.p.) or rHuEPO (100 U/kg, s.c.) given three times per week were studied in Dahl S rats fed an $8 \% \mathrm{NaCl}$ diet for 3 weeks. There was no difference in food intake or body weight among the groups (data not shown), indicating that these drugs were well tolerated. Hemoglobin and hematocrit levels in the groups treated with vehicle and $60 \mathrm{mg} / \mathrm{kg}$ of DMOG remained unchanged throughout the study. The hemoglobin and hematocrit levels in the group treated with $600 \mathrm{mg} / \mathrm{kg}$ of DMOG increased from $16.3 \pm 0.2 \mathrm{~g} / \mathrm{dl}$ and $56.1 \% \pm 2.0 \%$ to $18.3 \pm 0.4 \mathrm{~g} / \mathrm{dl}$ and $63.1 \% \pm 1.4 \%$ after 3 weeks of treatment. These values were comparable to the rise in hemoglobin and hematocrit levels seen in the rHuEPOtreated group $(18.8 \pm 0.4 \mathrm{~g} / \mathrm{dl}$ and $63.3 \% \pm 1.8 \%$, respectively $)$ (Fig. 2).

Effects of DMOG rHuEPO on the Development of Hypertension and Renal Injury. A comparison of the effects of DMOG or rHuEPO treatment on blood pressure and urinary protein excretion in Dahl S rats fed a high-salt diet is presented in Fig. 3. Baseline systolic blood pressure averaged $161 \pm 3 \mathrm{~mm} \mathrm{Hg}$ in the vehicle-treated group and rose to $226 \pm 4 \mathrm{~mm} \mathrm{Hg}$ after 3 weeks on a high-salt diet (Fig. 3A). Blood pressure and proteinuria were not different in the vehicle-treated group and the group treated with $60 \mathrm{mg} / \mathrm{kg}$ of DMOG (Fig. 3). Blood pressure and proteinuria increased to a greater extent in rats treated with $\mathrm{rHuEPO}$. Chronic treatment with $600 \mathrm{mg} / \mathrm{kg}$ of DMOG attenuated the development of hypertension and proteinuria, and systolic blood pressure only increased to $189 \pm 8 \mathrm{~mm} \mathrm{Hg}$ over the 3 -week course of the study.

Effects of DMOG and rHuEPO on Renal Injury. At baseline, glomerular injury and fibrosis were rare (Fig. 4, A and $\mathrm{B}$ ). The degree of glomerulosclerosis and fibrosis was markedly enhanced in the vehicle-treated group fed a highsalt diet for 3 weeks (Fig. 4C). They also exhibited some degree of renal interstitial fibrosis (Fig. 4D). The degree of glomerulosclerosis and renal interstitial fibrosis was markedly enhanced in rats receiving $\mathrm{rHuEPO}$ (Fig. 4, G and $\mathrm{H}$ ). In contrast, the degree of glomerulosclerosis and glomerular fibrosis, as well as renal interstitial fibrosis, was markedly reduced in rats treated with $600 \mathrm{mg} / \mathrm{kg}$ of DMOG (Fig. 4, E and F).

A comparison of the glomerulosclerosis score and degree of renal fibrosis is presented in Fig. 4, I-K. The glomerular sclerosis index and degree of glomerular fibrosis were similar in rats treated with vehicle versus rHuEPO. However, rHuEPO markedly enhanced the development of renal interstitial fibrosis. The degree of glomerulosclerosis and fibrosis and renal interstitial fibrosis was markedly attenuated in a dose-dependent manner in rats treated with DMOG.

A comparison of plasma BUN and serum creatinine levels among the groups is presented in Table 1. Plasma BUN and creatinine levels increased similarly in all the groups following 3 weeks on a high-salt diet. Urinary NAG and KIM-1 excretion increased following the development of hypertension in the vehicle-treated rats. rHuEPO increased urinary KIM-1 excretion to a greater extent than that seen in the vehicle-treated group. The increases in NAG and KIM-1 excretion were markedly attenuated in the rats treated with DMOG (Fig. 5).

The degree of fibrosis in the outer medulla of the kidney was markedly elevated in vehicle-treated rats fed a high-salt diet for 3 weeks. rHuEPO aggravated renal medullary fibrosis (Fig. 6G), whereas it was completely prevented in rats treated with DMOG (Fig. 6E). The formation of protein casts was increased dramatically following the development of hypertension in the vehicle-treated group (Fig. 6, B and D). DMOG, as well as rHuEPO, attenuated the formation of protein casts
A

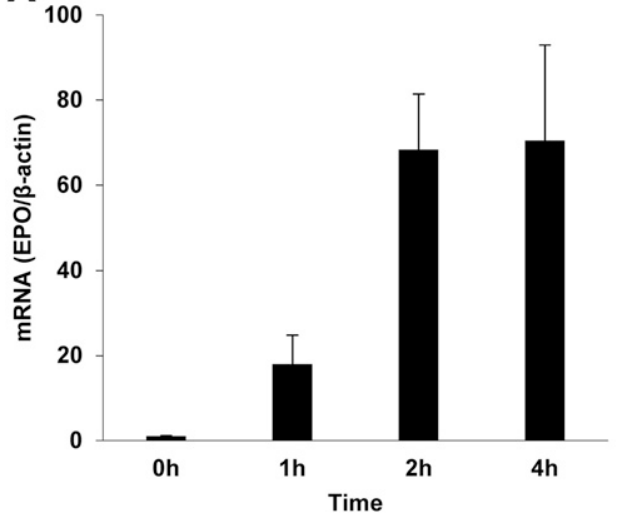

B

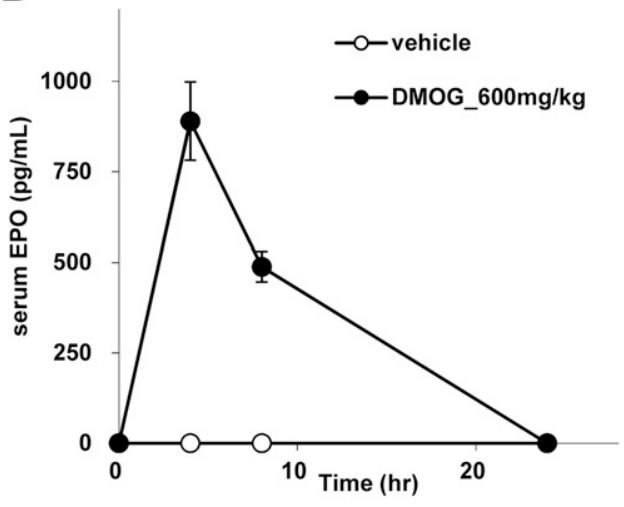

Fig. 1. Time-course study of the effects of DMOG $(600 \mathrm{mg} / \mathrm{kg})$ on EPO mRNA expression and serum EPO concentration. (A) DMOG increased EPO mRNA expression level in renal cortex. (B) Serum EPO concentration increased 4 hours after the DMOG administration and returned to the baseline level thereafter. Open circles represent serum EPO concentration in vehicle-treated animals, and closed circles represent levels in DMOG-treated animals. Data are represented as mean values \pm 1 S.E.M. from $n=3$ animals per group. 

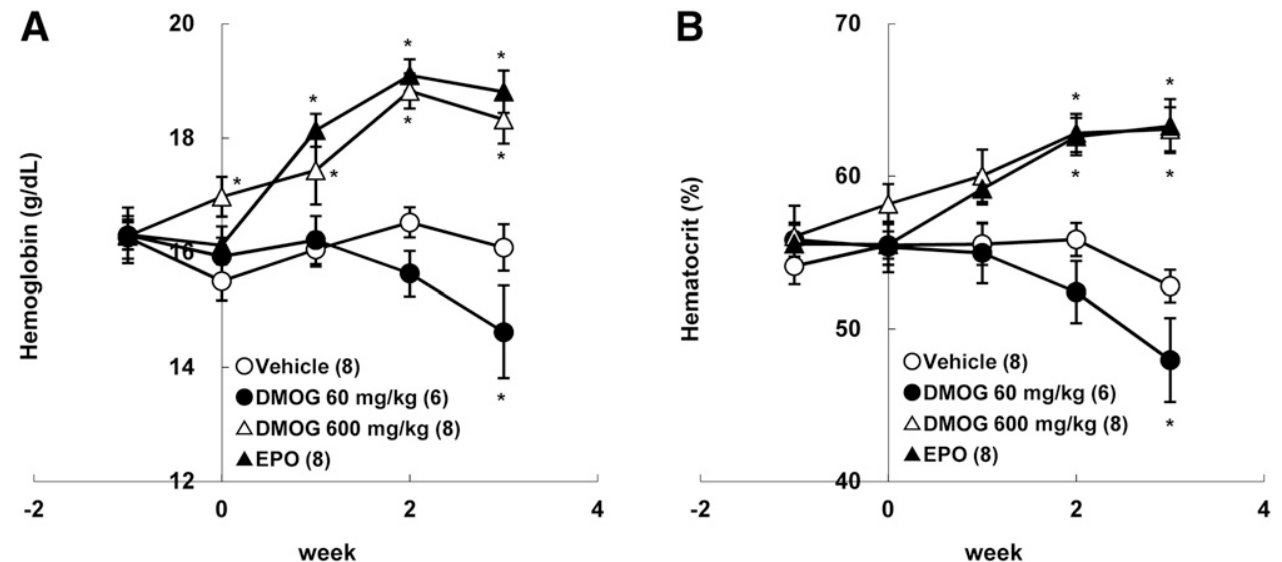

Fig. 2. Effect of DMOG and rHuEPO on (A) hemoglobin and (B) hematocrit in high-salt-fed Dahl S rats before and after chronic administration of vehicle (open circle), $60 \mathrm{mg} / \mathrm{kg}$ of DMOG (filled circle), $600 \mathrm{mg} / \mathrm{kg}$ of DMOG (open triangle), and $100 \mathrm{U} / \mathrm{kg}$ of rHuEPO (filled triangle). The high-salt diet and the drug administration were started together on day 1 after the control period. Hemoglobin and hematocrit were measured at 1 week before the start of the study through week 3 . Data are presented as the mean values \pm 1 S.E.M. The number of animals studied in each group is shown in parentheses. *, significant difference from the corresponding value in vehicle-treated rats.

(Fig. 6, F, H, and J). Quantitative analysis revealed that DMOG prevented the renal medullary fibrosis and protein cast formation in a dose-dependent manner (Fig. 6, I and J).

Effects of DMOG and rHuEPO on Renal Inflammatory Markers and Growth Factors. High-salt treatment increased renal cortical and medullary IL-1 $\beta$ and MCP-1 compared with baseline levels (Fig. 7A). rHuEPO had no significant effect on the increase in renal IL- $1 \beta$ and MCP-1 levels. In contrast, DMOG treatment prevented the increase of IL-1 $\beta$ and MCP-1 levels in a dose-dependent manner.

Exposure to a high-salt treatment increased TGF $\beta 1$ levels in the medulla in the vehicle-treated group, and rHuEPO further increased the TGF $\beta 1$ levels. DMOG treatment had no significant effect on the rise in TGF $\beta 1$ expression.

The levels of VEGF in both cortex and medulla fell following 3 weeks on a high-salt diet in the vehicle-treated group. rHuEPO had no effect on the fall in VEGF expression (Fig. 8). In contrast, DMOG dose dependently increased renal VEGF levels by 7-fold in cortex and 60-fold in medulla compared with values seen in the vehicle-treated group.

\section{Discussion}

rHuEPO is widely used to treat anemia and improves the quality of life in patients with CKD (Evans et al., 1990). Several studies have explored the effects of $\mathrm{rHuEPO}$ on renal function in a variety of experimental models of CKD (Lee et al., 2005; Katavetin et al., 2007; Toba et al., 2009; Cañadillas et al., 2010; Rjiba-Touati et al., 2012). In general, these studies indicate that $\mathrm{rHuEPO}$ reduced renal inflammation and fibrosis regardless of its hematopoietic effect. However, subsequent clinical trials indicate that the use of rHuEPO increased the risks of hypertension, stroke, cardiovascular events, and dialysis in patients with CKD (Drüeke et al., 2006; Singh et al., 2006; Szczech et al., 2008; Pfeffer et al., 2009). Because hypertension and diabetes are the primary risk factors for the development of CKD; it is possible that these patients may be more susceptible to potential adverse effects of rHuEPO on blood pressure and hypertension-induced renal injury. To explore this possibility, we compared the effects of rHuEPO and DMOG, an injectable PHD inhibitor, on the development of hypertension and renal injury in Dahl S rats, which is an experimental model that is highly susceptible to the development of salt-sensitive hypertension and renal injury.

Treatment of Dahl S rats fed a high-salt diet with $\mathrm{rHuEPO}$ not only increased hemoglobin levels but also augmented the degree of hypertension. The treatment of the rats with $\mathrm{rHuEPO}$ also aggravated proteinuria and fibrosis in the renal cortex and medulla. These effects were associated with increased expression of TGF $\beta 1$. These results are consistent with the clinical findings that stimulation of hematopoiesis by rHuEPO treatment in patients with CKD is associated with increased risk of hypertension and renal dysfunction (Raine, 1988; Drüeke et al., 2006). Interestingly, in other experimental models of renal disease, such as cyclosporine-induced nephropathy, unilateral ureter obstruction, and diabetic

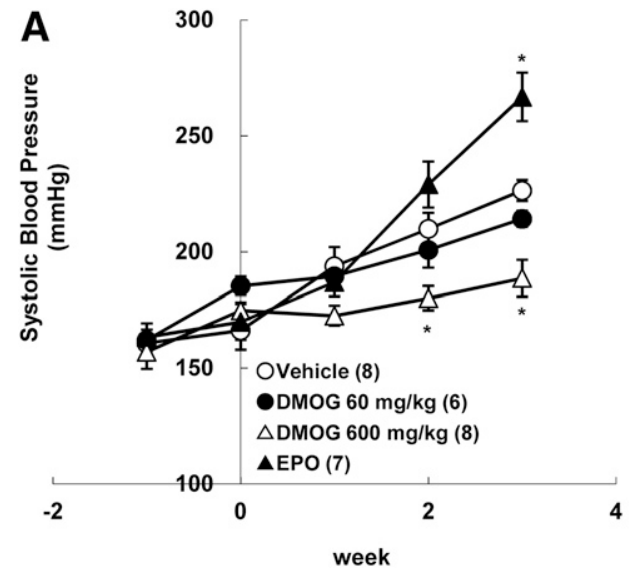

B

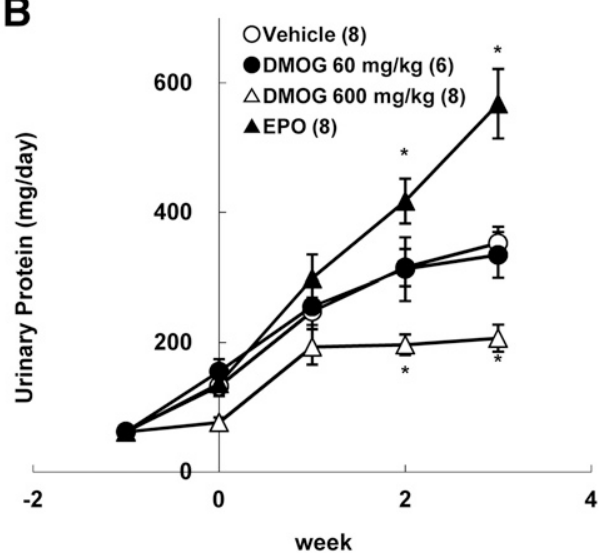

Fig. 3. Effect of DMOG and rHuEPO on (A) systolic blood pressure and (B) urinary protein excretion in Dahl $\mathrm{S}$ rats fed a high-salt diet before and after chronic administration of vehicle (open circle), $60 \mathrm{mg} / \mathrm{kg}$ of DMOG (filled circle), $600 \mathrm{mg} / \mathrm{kg}$ of DMOG (open triangle), and $100 \mathrm{U} / \mathrm{kg}$ of rHuEPO (filled triangle). The high-salt diet and the drug administration were started together on day 1 after the control period. The drugs were given three times per week. Systolic blood pressure and urinary protein excretion were measured at 1 week before the start of the study through week 3. Data are presented as the mean values \pm 1 S.E.M. The number of animals studied per group is shown in parentheses. *, significant difference from the corresponding value in vehicletreated rats. 

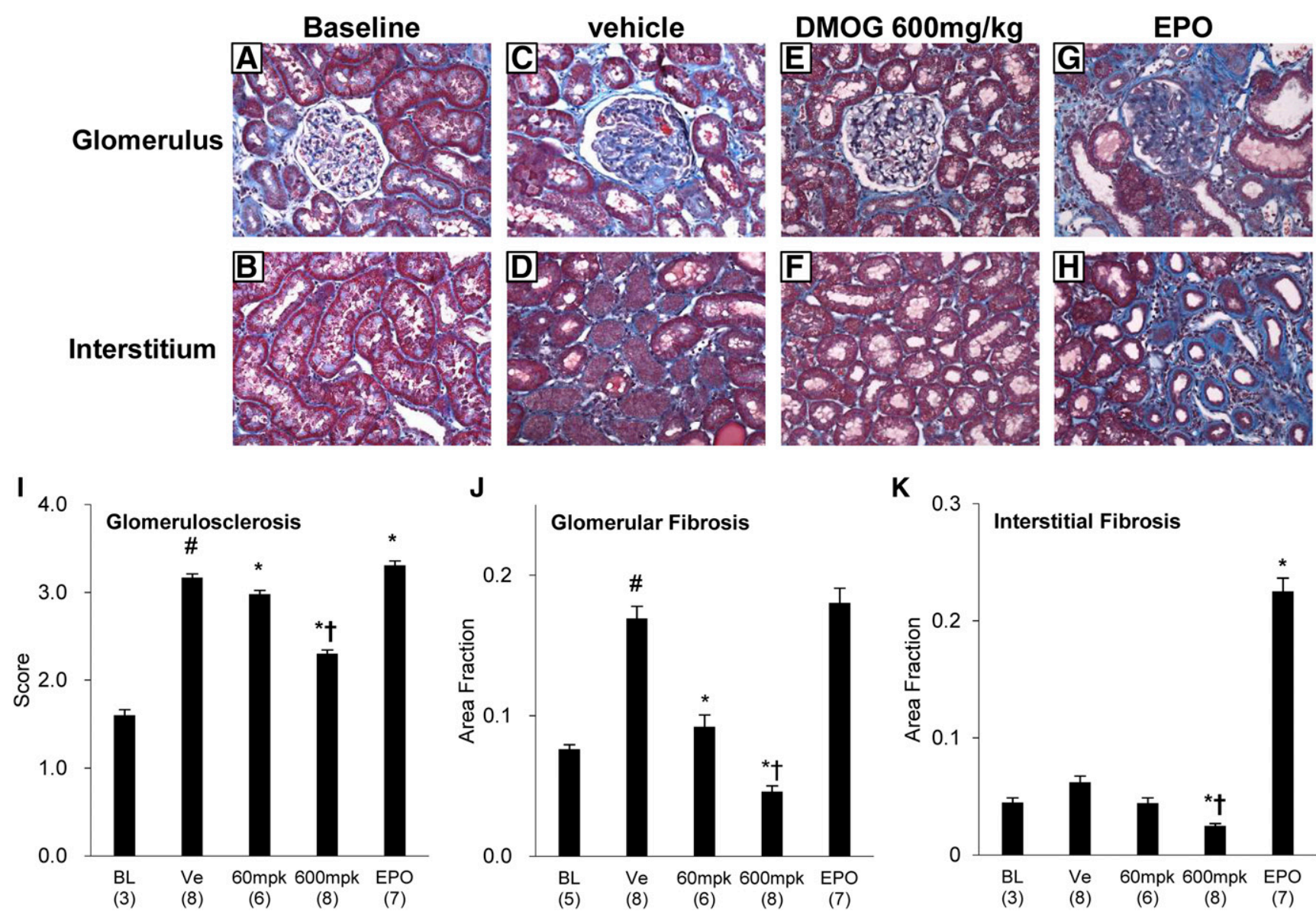

$\mathbf{J}$

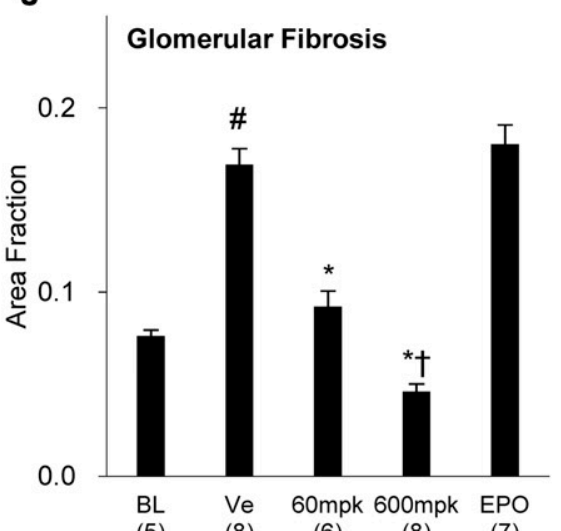

K

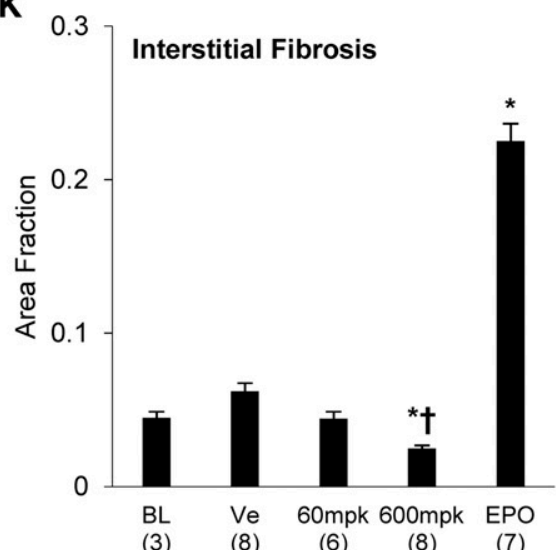

Fig. 4. Effect of DMOG and rHuEPO on glomerular injury and renal interstitial fibrosis in the renal cortex. Representative renal micrographs of the rats at baseline (A and B) and after treatment with vehicle (C and D), $600 \mathrm{mg} / \mathrm{kg}$ of DMOG (E and F), or rHuEPO (G and H) are shown. (A, C, E, and G) are micrographs of glomerulus, and (B, D, F, and H) are micrographs of the interstitium. Original magnification, 200×. Glomerulosclerosis (I), glomerular fibrosis $(\mathrm{J})$, and interstitial fibrosis $(\mathrm{K})$ are quantified and shown in the bar graphs. Thirty glomeruli were scored for the degree of glomerulosclerosis, and 10 fields were quantified for fibrosis in each animal. The number of animals studied per group is presented on the graphs. \#, significant difference from the corresponding baseline value; *, significant difference from the corresponding value in the vehicle-treated group; $\dagger$, significant difference from the corresponding value in the EPO-treated group; BL, baseline; Ve, vehicle.

nephropathy, and in normotensive strains of rats and mice that are not salt sensitive, blood pressure did not increase following administration of $\mathrm{rHuEPO}$. Moreover, $\mathrm{rHuEPO}$ suppressed TGF $\beta 1$ expression and reduced renal inflammation and fibrosis in several of these studies (Lee et al., 2005; Park et al., 2007; Toba et al., 2009). Because the blockage of TGF $\beta 1$ attenuates hypertension and renal fibrosis (Dahly et al., 2002; Murphy et al., 2012), these results suggest that in models in which rHuEPO does not increase blood pressure,

TABLE 1

BUN and serum creatinine

Mean values \pm 1 S.E.M. are presented.

\begin{tabular}{lcll}
\hline & $n$ & BUN $(\mathrm{mg} / \mathrm{dl})$ & sCre $(\mathrm{mg} / \mathrm{dl})$ \\
\hline Baseline & $8-10$ & $10.8 \pm 0.4$ & $0.56 \pm 0.02$ \\
Vehicle & 8 & $25.6 \pm 1.9^{*}$ & $1.07 \pm 0.12^{*}$ \\
DMOG & & & \\
$\quad 60 \mathrm{mg} / \mathrm{kg}$ & 6 & $31.6 \pm 3.2$ & $0.96 \pm 0.10$ \\
$\quad 600 \mathrm{mg} / \mathrm{kg}$ & 8 & $36.2 \pm 1.8$ & $0.89 \pm 0.03$ \\
$\begin{array}{l}\text { EPO } \\
100 \mathrm{U} / \mathrm{kg}\end{array}$ & 7 & $33.6 \pm 4.1$ & $0.97 \pm 0.05$ \\
\hline
\end{tabular}

*Indicates $P<0.05$ from the pooled baseline value. sCre, serum creatinine concentration.
rHuEPO can attenuate renal interstitial fibrosis by reducing $\mathrm{TGF} \beta 1$ expression. In contrast, our results indicate that when rHuEPO increased both hemoglobin levels and blood pressure, the rise in blood pressure may increase renal fibrosis.

DMOG is a PHD $1 / 2 / 3$ pan inhibitor that stimulates erythropoiesis by stabilizing HIFs (Epstein et al., 2001; Barrett et al., 2011). We compared the effects of rHuEPO and that of DMOG on blood pressure and renal function. In our experiments, $600 \mathrm{mg} / \mathrm{kg}$ of DMOG increased endogenous EPO concentration in serum and increased hemoglobin levels as potently as rHuEPO. However, in sharp contrast to rHuEPO, DMOG attenuated the development of hypertension and proteinuria in the Dahl S rats. Furthermore, DMOG prevented the increase of renal inflammatory markers in the kidney along with renal fibrosis and medullary protein cast formation. These results are consistent with the previous findings that the activation of HIF $1 \alpha$ attenuated the rise of blood pressure in high-salt-treated Dahl S rats (Zhu et al., 2012 , 2014). However, unlike the results of the previous study, we could not confirm an increase in heme oxygenase-1 expression in the kidney of Dahl S rats (data not shown). We instead found that renal VEGF levels were very low in highsalt-treated Dahl $\mathrm{S}$ rats and that it increased in the rats 

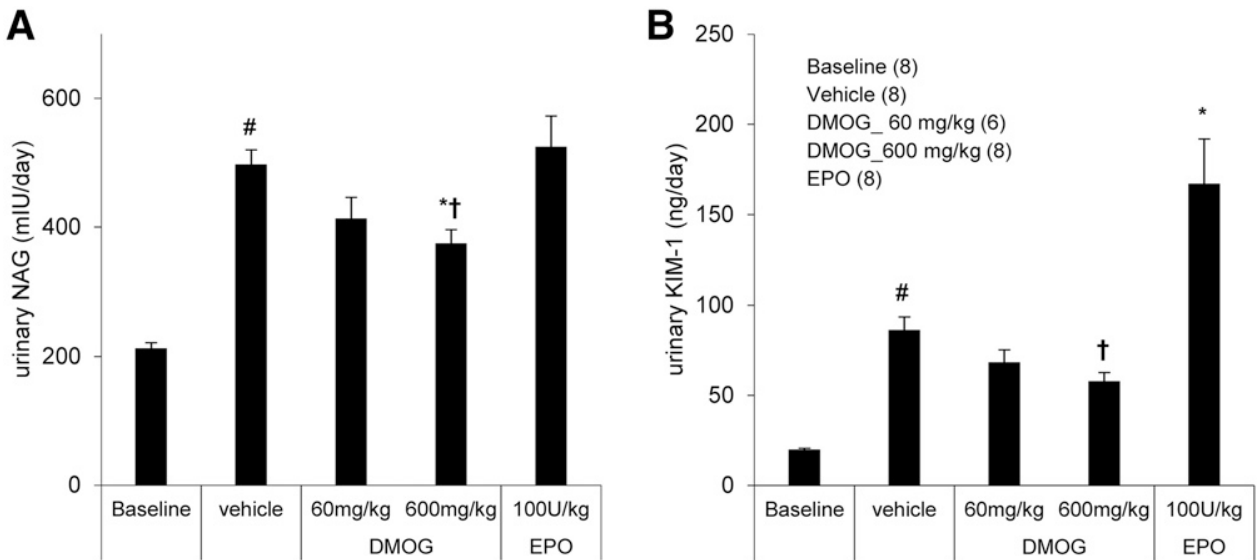

Fig. 5. Effect of DMOG and rHuEPO on (A) urinary NAG and (B) KIM-1 excretion in Dahl $\mathrm{S}$ rats fed a high-salt diet. The number of animals studied in each group is shown on the graphs. \#, significant difference from the corresponding baseline value; *, significant difference from the corresponding value in the vehicletreated group; $\dagger$, significant difference from the corresponding value in the EPO-treated group.

treated with DMOG. The renoprotective effects of VEGF have been reported elsewhere (Kang et al., 2001; Suga et al., 2001; Ma et al., 2011; Sivaskandarajah et al., 2012). Suppression of VEGF signaling using a humanized monoclonal antibody, genetic knockout of VEGF, or pharmacological blockade (Eremina et al., 2008; Lankhorst et al., 2017) has been shown to increase blood pressure. Interestingly, Eremina et al. (2008) found that glomerular injury preceeded the development of hypertension in VEGF knockout mice. Their observations are consistent with the present results that renal fibrosis and injury were attenuated in the group treated with $60 \mathrm{mg} / \mathrm{kg}$ of DMOG, whereas the hemoglobin levels and blood pressure
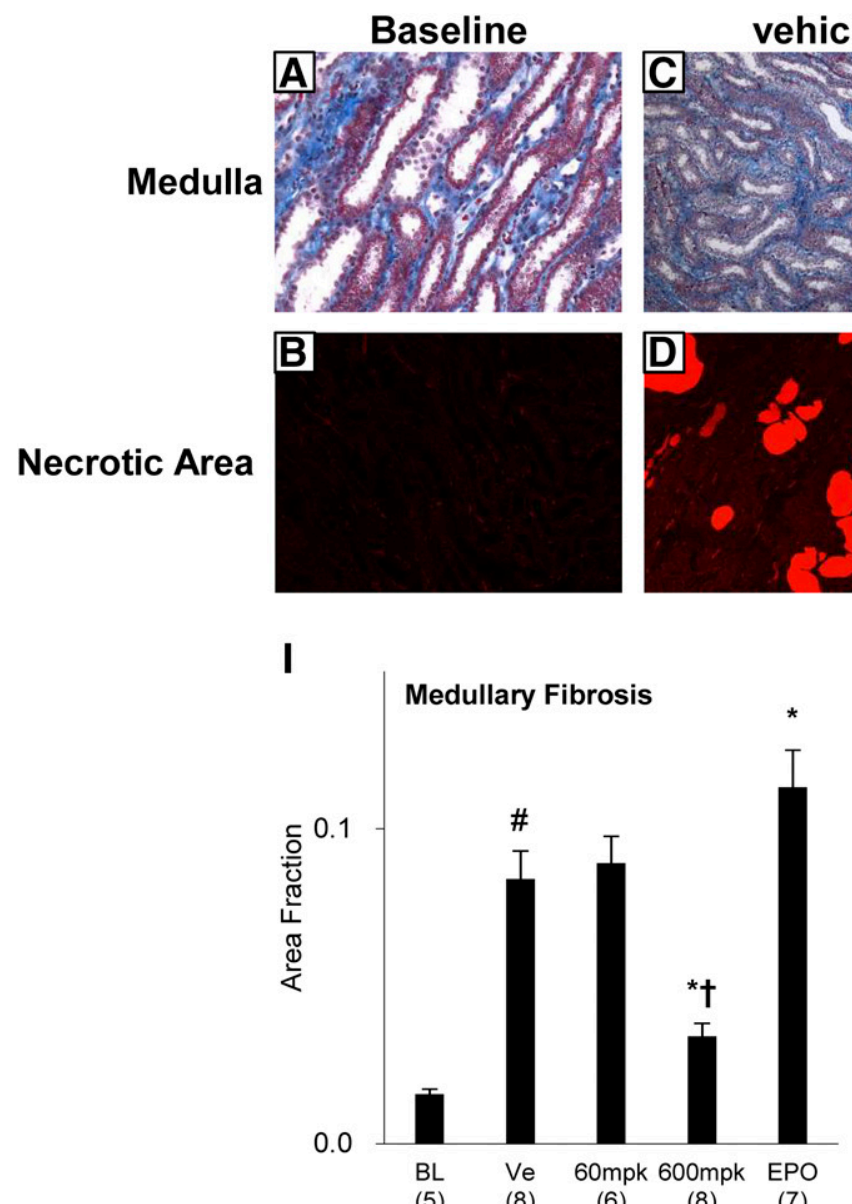
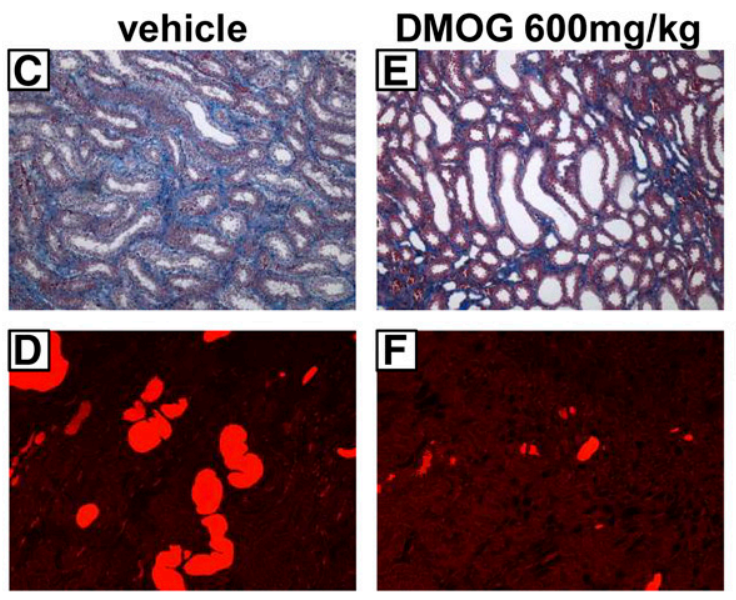

$\mathbf{J}$

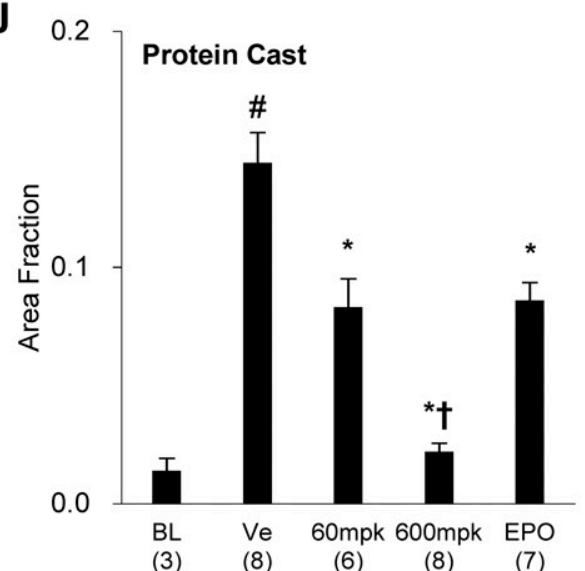

Fig. 6. Effect of DMOG and rHuEPO on renal injury in the medulla. Representative renal micrographs of the rats at baseline (A and B) and after treatment with vehicle (C and D), $600 \mathrm{mg} / \mathrm{kg}$ of DMOG (E and F), or $100 \mathrm{U} / \mathrm{kg}$ of rHuEPO (G and H) are shown. (A, C, E, and G) are micrographs of medullas, and (B, D, F, and H) are micrographs of protein casts. Original magnification, $100 \times$. Medullary fibrosis (I) and protein cast area (J) are quantified and shown in the bar graphs. Ten fields were quantified for fibrosis, and five fields were quantified for protein cast area in each animal. The number of animals studied per group are shown on the graphs. \#, significant difference from the corresponding baseline value; *, significant difference from the corresponding value in the vehicle-treated group; $\dagger$, significant difference from the corresponding value in the EPO-treated group; BL, baseline; Ve, vehicle. 
A
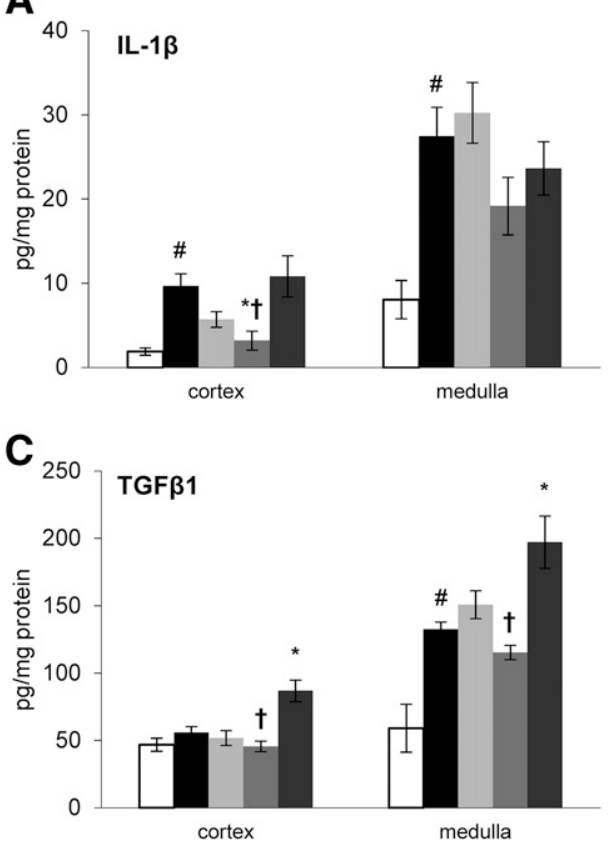

B

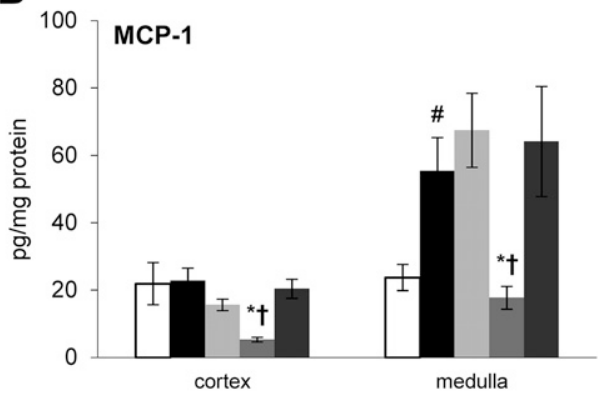

Fig. 7. Effect of DMOG and rHuEPO on (A) IL- $1 \beta$, (B) MCP-1, and (C) TGF $\beta 1$ levels in the renal cortex and medulla in Dahl $\mathrm{S}$ rats fed a high-salt diet. The number of animals studied per group is shown on the graphs. \#, significant difference from the corresponding baseline value; *, significant difference from the corresponding value in the vehicletreated group; $\dagger$, significant difference from the corresponding value in the EPO-treated group. were similar to the vehicle-treated group. Overall, these findings suggest that the reduction of renal VEGF expression could be one of the mechanisms contributing to the development of hypertension and renal injury in Dahl S rats. Based on this mechanism, DMOG could oppose the development of nephropathy by induction of renal VEGF expression and the attenuation of hypertension.

HIF1 $\alpha$ induces CD73 (ecto-5-prime-nucleotidase) (Synnestvedt et al., 2002) and increases adenosine signaling. In the kidney, adenosine is a vasoconstrictor involved in tubuloglomerular feedback responses (Schnermann, 2015; Romero and Carretero, 2019). Upregulation of CD73 has been reported to protect the kidney from ischemia-reperfusion injury and diabetic nephropathy (Tak et al., 2014; Sung et al., 2017). Therefore, PHD inhibitors may protect the kidney from saltsensitive hypertension and diabetes-induced renal injury by enhancing tubuloglomerular feedback responsiveness and reducing transmission of systemic pressure to the glomerulus.

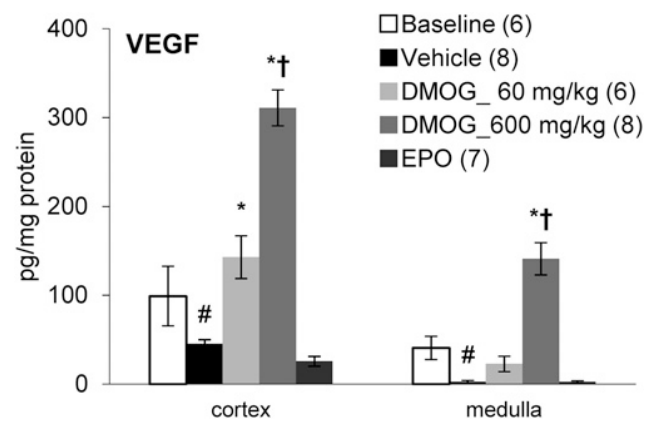

Fig. 8. Effect of DMOG and rHuEPO on VEGF expression in the renal cortex and medulla. The number of animals studied in each group is shown on the graphs. \#, significant difference from the corresponding baseline value; *, significant difference from the corresponding value in the vehicletreated group; $\dagger$, significant difference from the corresponding value in the EPO-treated group.
Currently, PHD inhibitors are under the investigation of phase 3 clinical trials to treat renal anemia in nondialysis and dialysis patients (Cernaro et al., 2019), and roxadustat was first approved for the treatment of anemia in China (Dhillon, 2019). The potential adverse events of three PHD inhibitorsmolidustat, daprodustat, and vadadustat-have been investigated in recent trials. Although the administration of molidustat was as effective as rHuEPO in increasing hemoglobin in patients with $\mathrm{CKD}$, the incidence of hypertension was lower in the molidustat-treated group (Macdougall et al., 2019). Daprodustat was also effective in increasing hemoglobin levels by more than $2 \mathrm{~g} / \mathrm{dl}$, but it did not affect blood pressure (Brigandi et al., 2016). Additionally, vadadustat increased hemoglobin levels by $1.4 \mathrm{~g} / \mathrm{dl}$ without altering blood pressure rise in a 6-week study (Martin et al., 2017); however, the number of patients with hypertension was higher in the vadadustat-treated group in a 20 -week study (Pergola et al., 2016). Although the statistical power in these clinical trials was insufficient to detect the change in blood pressure, it is noteworthy that the results from these clinical trials suggest that treatment of anemia with PHD inhibitors did not raise blood pressure. In the present study, we found that the blood pressure was lower in the DMOG-treated group than in the vehicle-treated group. This result is consistent with the results of the previous clinical trials and contrasts with the prohypertensive effects seen in rats treated with $\mathrm{rHuEPO}$.

DMOG has been reported to increase VEGF expression in the eye (Safran et al., 2006). Thus, there is a risk of retinopathy. However, in the present study, we did not observe any adverse effects in the Dahl S rats chronically treated with 60 or $600 \mathrm{mg} / \mathrm{kg}$ per day of DMOG.

In summary, rHuEPO and DMOG are equally effective in increasing hemoglobin levels in Dahl S rats fed a high-salt diet. However, rHuEPO aggravated the degree of hypertension, proteinuria, and renal injury. This was associated with induction of the expression of TGF $\beta 1$. In contrast, DMOG attenuated the development of hypertension and renal injury 
through induction of renal VEGF expression. These results suggest that PHD inhibitors may provide an alternative and safer therapeutic option for the treatment of anemia in patients with diabetes- and hypertension-induced CKD.

\section{Acknowledgments}

The authors thank Dr. Naoki Kojima, Dr. Yoshikazu Muroya, and Dr. Takekazu Kubo for their assistance and helpful comments on this manuscript.

\section{Authorship Contributions}

Participated in research design: Kato, Takahashi, Miyata, Roman. Conducted experiments: Kato.

Performed data analysis: Kato, Roman.

Wrote or contributed to the writing of the manuscript: Kato, Roman.

\section{References}

Asada N, Takase M, Nakamura J, Oguchi A, Asada M, Suzuki N, Yamamura K, Nagoshi N, Shibata S, Rao TN, et al. (2011) Dysfunction of fibroblasts of extrarenal origin underlies renal fibrosis and renal anemia in mice. J Clin Invest 121: 3981-3990.

Barrett TD, Palomino HL, Brondstetter TI, Kanelakis KC, Wu X, Haug PV, Yan W, Young A, Hua H, Hart JC, et al. (2011) Pharmacological characterization of 1-(5chloro-6-(trifluoromethoxy)-1H-benzoimidazol-2-yl)-1H-pyrazole-4-carboxylic acid (JNJ-42041935), a potent and selective hypoxia-inducible factor prolyl hydroxylase inhibitor. Mol Pharmacol 79:910-920.

Brigandi RA, Johnson B, Oei C, Westerman M, Olbina G, de Zoysa J, Roger SD, Sahay M, Cross N, McMahon L, et al.; PHI112844 Investigators (2016) A novel hypoxia-inducible factor-prolyl hydroxylase inhibitor (GSK1278863) for anemia in CKD: a 28-day, phase 2A randomized trial. Am J Kidney Dis 67:861-871.

Cañadillas S, Ortega R, Estepa JC, Egea J, Gonzalez-Menchen A, Perez-Seoane C, Lopez-Andreu M, Ramirez R, Tetta C, Rodriguez M, et al. (2010) Darbepoetin- $\alpha$ treatment enhances glomerular regenerative process in the Thy-1 glomerulonephritis model. Am J Physiol Renal Physiol 299:F1278-F1287.

Cernaro V, Coppolino G, Visconti L, Rivoli L, Lacquaniti A, Santoro D, Buemi A, Loddo S, and Buemi M (2019) Erythropoiesis and chronic kidney disease-related anemia: from physiology to new therapeutic advancements. Med Res Rev $\mathbf{3 9}$ 427-460.

Dahly AJ, Hoagland KM, Flasch AK, Jha S, Ledbetter SR, and Roman RJ (2002) Antihypertensive effects of chronic anti-TGF-beta antibody therapy in Dahl S rats Am J Physiol Regul Integr Comp Physiol 283:R757-R767.

Dhillon S (2019) Roxadustat: first global approval. Drugs 79:563-572.

Drüeke TB, Locatelli F, Clyne N, Eckardt KU, Macdougall IC, Tsakiris D, Burger HU, and Scherhag A; CREATE Investigators (2006) Normalization of hemoglobin level in patients with chronic kidney disease and anemia. $N$ Engl J Med 355 : 2071-2084.

Elson DA, Thurston G, Huang LE, Ginzinger DG, McDonald DM, Johnson RS, and Arbeit JM (2001) Induction of hypervascularity without leakage or inflammation in transgenic mice overexpressing hypoxia-inducible factor-1alpha Genes Dev 15:2520-2532.

Epstein AC, Gleadle JM, McNeill LA, Hewitson KS, O'Rourke J, Mole DR, Mukherji M, Metzen E, Wilson MI, Dhanda A, et al. (2001) C. elegans EGL-9 and mammalian homologs define a family of dioxygenases that regulate HIF by prolyl hydroxylation. Cell 107:43-54.

Eremina V, Jefferson JA, Kowalewska J, Hochster H, Haas M, Weisstuch J, Richardson C, Kopp JB, Kabir MG, Backx PH, et al. (2008) VEGF inhibition and renal thrombotic microangiopathy. N Engl J Med 358:1129-1136.

Evans RW, Rader B, and Manninen DL; Cooperative Multicenter EPO Clinical Trial Group (1990) The quality of life of hemodialysis recipients treated with recombinant human erythropoietin. JAMA 263:825-830.

Flamme I, Oehme F, Ellinghaus P, Jeske M, Keldenich J, and Thuss U (2014) Mimicking hypoxia to treat anemia: HIF-stabilizer BAY 85-3934 (Molidustat) stimulates erythropoietin production without hypertensive effects. PLoS One $\mathbf{9}$ e111838

Haase VH (2006) Hypoxia-inducible factors in the kidney. Am J Physiol Renal Physiol 291:F271-F281.

Jaakkola P, Mole DR, Tian YM, Wilson MI, Gielbert J, Gaskell SJ, von Kriegsheim A, Hebestreit HF, Mukherji M, Schofield CJ, et al. (2001) Targeting of HIF-alpha to the von Hippel-Lindau ubiquitylation complex by O2-regulated prolyl hydroxylation. Science 292:468-472.

Kang DH, Hughes J, Mazzali M, Schreiner GF, and Johnson RJ (2001) Impaired angiogenesis in the remnant kidney model: II. Vascular endothelial growth factor administration reduces renal fibrosis and stabilizes renal function. $J$ Am Soc Nephrol 12:1448-1457.

Katavetin P, Inagi R, Miyata T, Shao J, Sassa R, Adler S, Eto N, Kato H, Fujita T, and Nangaku M (2007) Erythropoietin induces heme oxygenase-1 expression and attenuates oxidative stress. Biochem Biophys Res Commun 359:928-934.

Kato S, Takayama N, Takano H, Koretsune H, Koizumi C, Kunioka EI, Uchida S, Takahashi T, and Yamamoto K (2018) TP0463518, a novel inhibitor for hypoxiainducible factor prolyl hydroxylases, increases erythropoietin in rodents and monkeys with a good pharmacokinetics-pharmacodynamics correlation. Eur $J$ Pharmacol 838:138-144.

Koury MJ and Haase VH (2015) Anaemia in kidney disease: harnessing hypoxia responses for therapy. Nat Rev Nephrol 11:394-410.
Kuhrt D and Wojchowski DM (2015) Emerging EPO and EPO receptor regulators and signal transducers. Blood 125:3536-3541.

Lankhorst S, Severs D, Markó L, Rakova N, Titze J, Müller DN, Danser AH, and van den Meiracker AH (2017) Salt sensitivity of angiogenesis inhibition-induced blood pressure rise: role of interstitial sodium accumulation? Hypertension 69:919-926.

Lee SH, Li C, Lim SW, Ahn KO, Choi BS, Kim YS, Moon IS, Kim J, Bang BK, and Yang CW (2005) Attenuation of interstitial inflammation and fibrosis by recombinant human erythropoietin in chronic cyclosporine nephropathy. Am J Nephrol 25:64-76.

Letcher RL, Chien S, Pickering TG, Sealey JE, and Laragh JH (1981) Direct relationship between blood pressure and blood viscosity in normal and hypertensive subjects. Role of fibrinogen and concentration. Am J Med 70:1195-1202.

Ma J, Matsusaka T, Yang HC, Zhong J, Takagi N, Fogo AB, Kon V, and Ichikawa I (2011) Induction of podocyte-derived VEGF ameliorates podocyte injury and subsequent abnormal glomerular development caused by puromycin aminonucleoside. Pediatr Res 70:83-89.

Macdougall IC, Akizawa T, Berns JS, Bernhardt T, and Krueger T (2019) Effects of molidustat in the treatment of anemia in CKD. Clin J Am Soc Nephrol 14:28-39. Martin ER, Smith MT, Maroni BJ, Zuraw QC, and deGoma EM (2017) Clinical trial of vadadustat in patients with anemia secondary to stage 3 or 4 chronic kidney disease. Am J Nephrol 45:380-388.

McFarlane SI, Chen SC, Whaley-Connell AT, Sowers JR, Vassalotti JA, Salifu MO, Li S, Wang C, Bakris G, McCullough PA, et al.; Kidney Early Evaluation Program Investigators (2008) Prevalence and associations of anemia of CKD: Kidney Early Evaluation Program (KEEP) and National Health and Nutrition Examination Survey (NHANES) 1999-2004. Am J Kidney Dis 51 (4):S46-S55.

Means RT Jr (2016) Pure red cell aplasia. Blood 128:2504-2509.

Muroya Y, Fan F, Regner KR, Falck JR, Garrett MR, Juncos LA, and Roman RJ (2015) Deficiency in the formation of 20-hydroxyeicosatetraenoic acid enhances renal ischemia-reperfusion injury. J Am Soc Nephrol 26:2460-2469.

Murphy SR, Dahly-Vernon AJ, Dunn KM, Chen CC, Ledbetter SR, Williams JM, and Roman RJ (2012) Renoprotective effects of anti-TGF- $\beta$ antibody and antihypertensive therapies in Dahl S rats. Am J Physiol Regul Integr Comp Physiol 303: R57-R69.

Obara N, Suzuki N, Kim K, Nagasawa T, Imagawa S, and Yamamoto M (2008) Repression via the GATA box is essential for tissue-specific erythropoietin gene expression. Blood 111:5223-5232.

Paliege A, Rosenberger C, Bondke A, Sciesielski L, Shina A, Heyman SN, Flippin LA, Arend M, Klaus SJ, and Bachmann S (2010) Hypoxia-inducible factor-2alphaexpressing interstitial fibroblasts are the only renal cells that express erythropoietin under hypoxia-inducible factor stabilization. Kidney Int 77:312-318.

Park SH, Choi MJ, Song IK, Choi SY, Nam JO, Kim CD, Lee BH, Park RW, Park KM, Kim YJ, et al. (2007) Erythropoietin decreases renal fibrosis in mice with ureteral obstruction: role of inhibiting TGF-beta-induced epithelial-to-mesenchymal transition. J Am Soc Nephrol 18:1497-1507.

Percy MJ, Furlow PW, Lucas GS, Li X, Lappin TR, McMullin MF, and Lee FS (2008) A gain-of-function mutation in the HIF2A gene in familial erythrocytosis. $N$ Engl $J$ Med 358:162-168.

Pergola PE, Spinowitz BS, Hartman CS, Maroni BJ, and Haase VH (2016) Vadadustat, a novel oral HIF stabilizer, provides effective anemia treatment in nondialysis-dependent chronic kidney disease. Kidney Int 90:1115-1122.

Pfeffer MA, Burdmann EA, Chen CY, Cooper ME, de Zeeuw D, Eckardt KU, Feyzi JM, Ivanovich P, Kewalramani R, Levey AS, et al.; TREAT Investigators (2009) A trial of darbepoetin alfa in type 2 diabetes and chronic kidney disease. $N$ Engl $J$ Med 361:2019-2032.

Raine AE (1988) Hypertension, blood viscosity, and cardiovascular morbidity in renal failure: implications of erythropoietin therapy. Lancet 1:97-100.

Rjiba-Touati K, Ayed-Boussema I, Bouaziz C, Belarbia A, Azzabi A, Achour A, Hassen W, and Bacha H (2012) Protective effect of erythropoietin against cisplatininduced nephrotoxicity in rats: antigenotoxic and antiapoptotic effect. Drug Chem Toxicol 35:89-95.

Romero CA and Carretero OA (2019) Tubule-vascular feedback in renal autoregulation. Am J Physiol Renal Physiol 316:F1218-F1226.

Safran M, Kim WY, O'Connell F, Flippin L, Günzler V, Horner JW, Depinho RA and Kaelin WG Jr (2006) Mouse model for noninvasive imaging of HIF prolyl hydroxylase activity: assessment of an oral agent that stimulates erythropoietin production. Proc Natl Acad Sci USA 103:105-110.

Schnermann J (2015) Concurrent activation of multiple vasoactive signaling pathways in vasoconstriction caused by tubuloglomerular feedback: a quantitative assessment. Annu Rev Physiol 77:301-322.

Singh AK, Szczech L, Tang KL, Barnhart H, Sapp S, Wolfson M, and Reddan D; CHOIR Investigators (2006) Correction of anemia with epoetin alfa in chronic kidney disease. N Engl J Med 355:2085-2098.

Sivaskandarajah GA, Jeansson M, Maezawa Y, Eremina V, Baelde HJ, and Quaggin $\mathrm{SE}$ (2012) Vegfa protects the glomerular microvasculature in diabetes. Diabetes $\mathbf{6 1}$ $2958-2966$.

Souma T, Yamazaki S, Moriguchi T, Suzuki N, Hirano I, Pan X, Minegishi N, Abe M, Kiyomoto H, Ito S, et al. (2013) Plasticity of renal erythropoietin-producing cells governs fibrosis. J Am Soc Nephrol 24:1599-1616.

Steffen HM, Brunner R, Müller R, Degenhardt S, Pollok M, Lang R, and Baldamus CA (1989) Peripheral hemodynamics, blood viscosity, and the renin-angiotensin system in hemodialysis patients under therapy with recombinant human erythropoietin. Contrib Nephrol 76:292-298.

Suga S, Kim YG, Joly A, Puchacz E, Kang DH, Jefferson JA, Abraham JA, Hughes J, Johnson RJ, and Schreiner GF (2001) Vascular endothelial growth factor (VEGF121) protects rats from renal infarction in thrombotic microangiopathy. Kidney Int 60:1297-1308.

Sung SJ, Li L, Huang L, Lawler J, Ye H, Rosin DL, Vincent IS, Le TH, Yu J, Görldt $\mathrm{N}$, et al. (2017) Proximal tubule CD73 is critical in renal ischemia-reperfusion injury protection. J Am Soc Nephrol 28:888-902. 
Synnestvedt K, Furuta GT, Comerford KM, Louis N, Karhausen J, Eltzschig HK, Hansen KR, Thompson LF, and Colgan SP (2002) Ecto-5'-nucleotidase (CD73) regulation by hypoxia-inducible factor- 1 mediates permeability changes in intestinal epithelia. J Clin Invest 110:993-1002.

Szczech LA, Barnhart HX, Inrig JK, Reddan DN, Sapp S, Califf RM, Patel UD, and Singh AK (2008) Secondary analysis of the CHOIR trial epoetin-alpha dose and achieved hemoglobin outcomes. Kidney Int 74:791-798.

Tak E, Ridyard D, Kim JH, Zimmerman M, Werner T, Wang XX, Shabeka U, Seo SW, Christians U, Klawitter J, et al. (2014) CD73-dependent generation of adenosine and endothelial Adora2b signaling attenuate diabetic nephropathy. J Am Soc Nephrol 25:547-563.

Toba H, Sawai N, Morishita M, Murata S, Yoshida M, Nakashima K, Morita Y, Kobara M, and Nakata T (2009) Chronic treatment with recombinant human erythropoietin exerts renoprotective effects beyond hematopoiesis in streptozotocin-induced diabetic rat. Eur J Pharmacol 612:106-114.

Zhu Q, Hu J, Han WQ, Zhang F, Li PL, Wang Z, and Li N (2014) Silencing of HIF prolyl-hydroxylase 2 gene in the renal medulla attenuates salt-sensitive hypertension in Dahl S rats. Am J Hypertens 27:107-113.

Zhu Q, Wang Z, Xia M, Li PL, Zhang F, and Li N (2012) Overexpression of HIF-1a transgene in the renal medulla attenuated salt sensitive hypertension in Dahl S rats. Biochim Biophys Acta 1822:936-941.

Address correspondence to: Dr. Richard J. Roman, Department of Pharmacology and Toxicology, University of Mississippi Medical Center, 2500 North State Street, Jackson, MS 39216. E-mail: rroman@umc.edu 